\title{
Gamma-ray burst precursors as observed by Fermi-GBM
}

\author{
Paul Coppin, ${ }^{a, *}$ Krijn D. de $\operatorname{Vries}^{a}$ and Nick van Eijndhoven ${ }^{a}$ \\ ${ }^{a}$ Vrije Universiteit Brussel, Dienst ELEM, \\ Pleinlaan 2, 1050 Brussels, Belgium \\ E-mail: paulcppn@gmail.com
}

Gamma-ray bursts (GRBs) are the most powerful outbursts of electromagnetic radiation in our Universe. A subset of GRBs are accompanied by precursors, dim gamma-ray flashes that precede the main outburst by tens to hundreds of seconds. We present an analysis of 11 years of FermiGBM data to identify these precursor flashes. For each of the 2364 analyzed GRBs, a time window of $2000 \mathrm{~s}$ centered on the GRB trigger was examined using a Bayesian block method. 217 GRBs (9\%) with precursor emission were identified. Our results indicate that long bursts ( $>2 \mathrm{~s}$ ) are $\sim 10$ times more likely to be preceded by a precursor than short bursts. In addition, we show that the distribution of the quiescent time, separating the precursor and the prompt (main emission) phase, is well modeled by a double Gaussian function. This suggests that at least two physical mechanisms contribute to the observed precursor flashes. One noteworthy GRB with precursor emission in our sample is the ultra-bright GRB 190114C, for which TeV gamma rays were observed by the MAGIC telescope. Our results, including the emission times and light curves of the identified precursors, have been made available via the GRBweb online tool (https://icecube.wisc.edu/ grbweb_public/Precursors.html).

$37^{\text {th }}$ International Cosmic Ray Conference (ICRC 2021)

July 12th - 23rd, 2021

Online - Berlin, Germany

\footnotetext{
*Presenter
} 


\section{Introduction}

Gamma-ray bursts (GRBs) were first discovered in 1967, just over half a century ago. They can be caused by two types of progenitors. Long bursts, generally lasting in excess of $2 \mathrm{~s}$ and in some extreme cases up to thousands of seconds, are thought to be triggered by the core collapse of a super-massive star. Short bursts, whose duration is generally less than $2 \mathrm{~s}$ down do tens of milliseconds, are expected to be caused by the merger of a neutron star with another compact object. Both types of progenitors are hypothesised to produce a black hole that launches a highly relativistic jet, accelerating particles and leading to the observed gamma-ray flux.

Since 1967, a wealth of observational evidence has been gathered on GRBs. They are one of the few source classes that have been imaged over the full electromagnetic spectrum, from radio all the way up to TeV gamma rays. So far, one burst (GRB 170817A) has also been detected by means of gravitational waves [1]. GRB observations can be classified in three stages. Prompt observations relate to the gamma rays and contemporary emission released at the time of the burst. This is followed by an afterglow phase, in which the material ejected by the jet remains visible for hours up to weeks at increasingly longer wavelengths. A subset of bursts also exhibit a third emission stage, called the GRB precursor. As the name suggests, precursors occur before the prompt phase, typically on the order of tens of seconds. They are observed as a slight increase in the gamma-ray emission, but dimmer than that of the prompt phase. Depending on the exact definition, $3 \%$ to $20 \%$ of GRBs are estimated to have a precursor [2-16].

Out of the three emission stages, precursors remain the least understood. Various models have been proposed, but there is currently no consensus on their origin. In this proceeding, we report and elaborate on the results of a systematic study, described in full detail in [16], that identified precursor emission in the light curves of Fermi-GBM bursts. Apart from characterising observational properties of GRB precursors, the timing data also allowed for multi-messenger follow-up studies. One temporal coincidence analysis by the IceCube collaboration, that searched for coincident neutrino events, has already been performed [17].

\section{Fermi-GBM data}

Currently, the Fermi Gamma-ray Burst Monitor (GBM) is the most prolific GRB detector in operation. Since its launch in 2008, it has been detecting an average of 300 bursts per year [18]. GBM itself consists of several subdetectors. Its main instruments are twelve identical sodium iodide (NaI) detectors that are pointing at different locations of the sky. GBM also houses two bismuth germanate (BGO) detectors that are sensitive to higher energies ${ }^{1}$. The BGO detectors mainly serve to cover the energy gap with the Fermi-LAT telescope and do not contribute to the triggering system of GBM. For the same reason, the analysis presented here likewise discards the BGO data, focusing on data from the two or three NaI subdetectors that were triggered by and pointing most closely at the burst. Up to 2020, Fermi-GBM observed 2684 bursts. For each of those GRBs a time interval of $2000 \mathrm{~s}$, centred on the GBM trigger time, is examined.

${ }^{1}$ The $\mathrm{NaI}$ and $\mathrm{BGO}$ detectors are sensitive to photons in the energy range from $8 \mathrm{keV}$ to $1 \mathrm{MeV}$ and $200 \mathrm{keV}$ to $40 \mathrm{MeV}$, respectively. Fermi-LAT is sensitive from $30 \mathrm{MeV}$ to $300 \mathrm{GeV}$ [18]. 


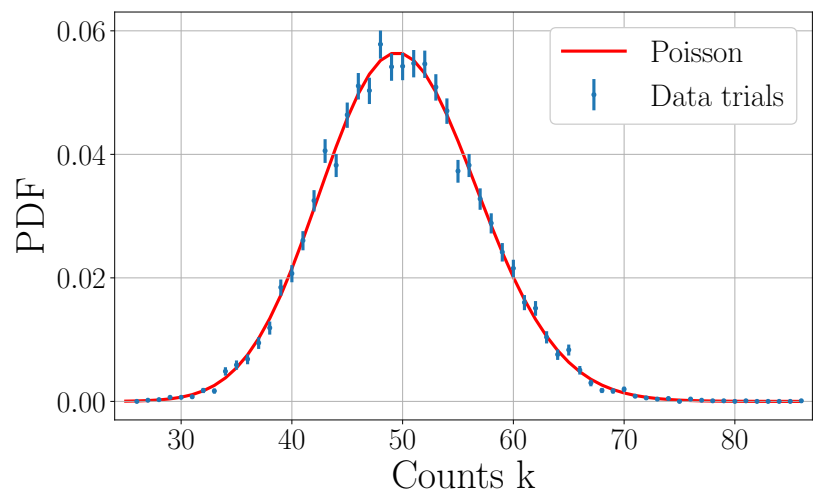

Figure 1: The rate $\lambda$ averaged over a $10 \mathrm{~s}$ period was calculated for background data of individual NaI detectors. By then plotting the distribution of photon counts $k$ observed in a time interval $50 \lambda^{-1}$ next to the Poisson expectation, we demonstrate that the background rate is well described by Poisson fluctuations.

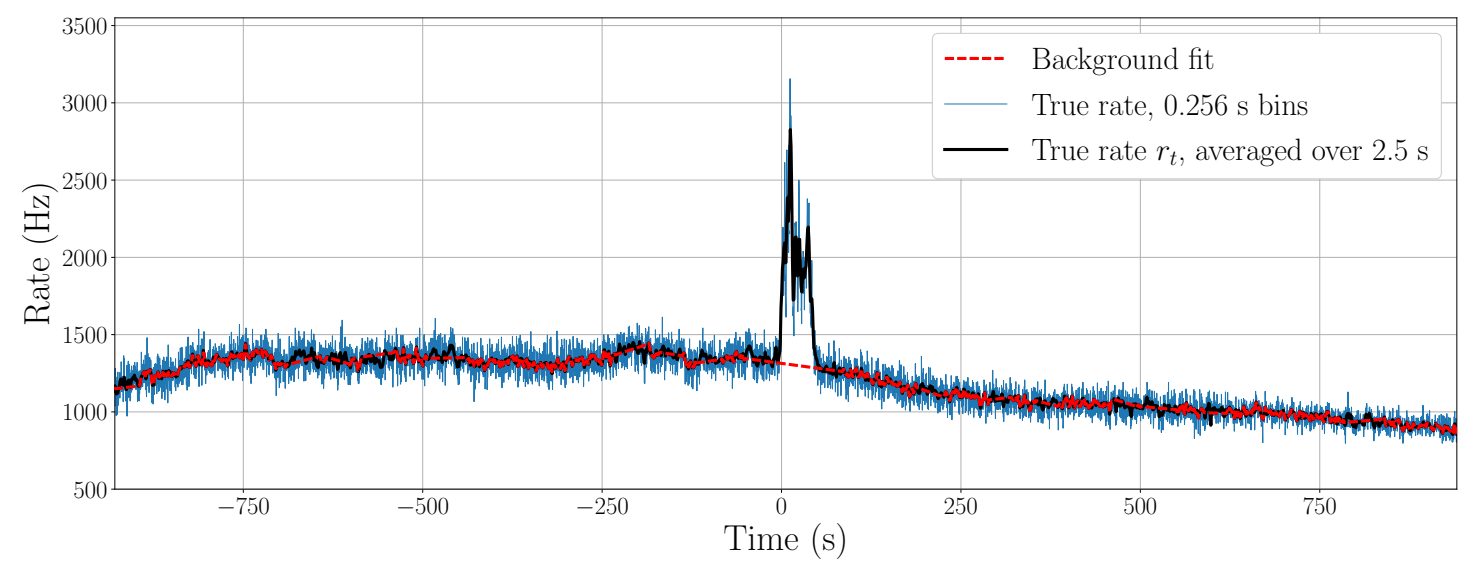

Figure 2: Illustration of the background rate characterisation procedure for GRB trigger bn 150422703 and the GBM subdetector labelled 'nb'.

\section{Analysis method}

At any given time, the individual $\mathrm{NaI}$ subdetectors each observe a typical background rate of the order $\sim 1 \mathrm{kHz}$. Figure 1 illustrates that on timescales smaller than a few tens of seconds, the background rate is well described by Poisson fluctuations. On longer timescales, the average rate slowly varies due to changes in the position and orientation of the satellite [19]. An example light curve, illustrating this behaviour, is shown in Fig. 2. To select the time regions that will be used to characterize the background rate, our analysis uses a novel approach inspired by the GBM online trigger. A linear fit over a small time range $[t-30 \mathrm{~s}, t-10 \mathrm{~s}]$ is used to predict what the rate at time $t$ will be. If the predicted rate then matches the actual observed rate, implying that there were no sudden changes, the rate at time $t$ will be used to characterize the background. This will, at a later stage in the analysis, allow us to construct a background subtracted light curve. A detailed description of this method is given in [16]. We here simply demonstrate the result. For the light curve shown in Fig. 2, the estimated background rate is depicted by the dashed red line. 


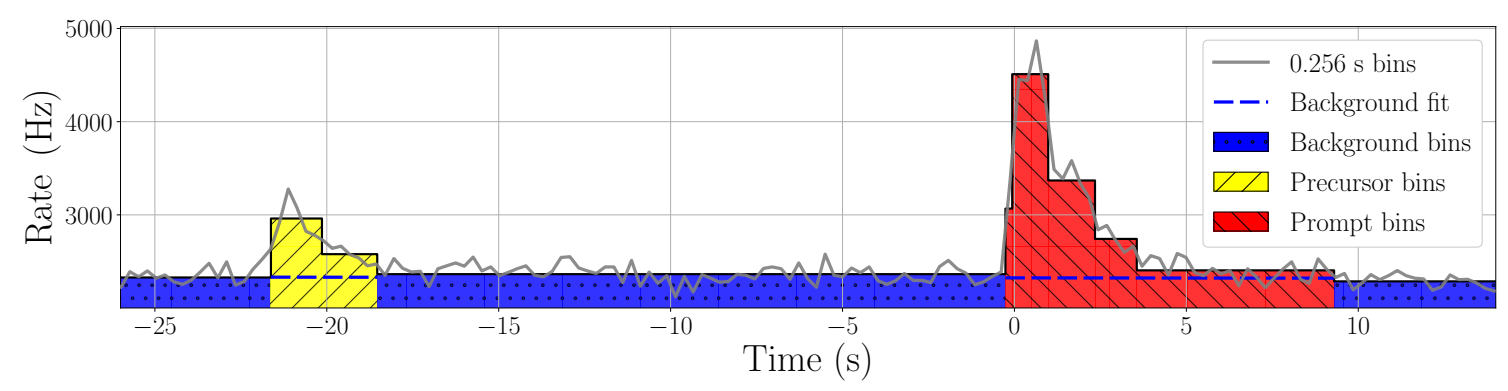

Figure 3: Light curve for GRB 120308588, illustrating the Bayesian block procedure.

The next stage in the analysis is to identify the exact moments in time at which the rate does undergo sudden changes, e.g. due to the appearance and subsequent disappearance of a signal component. The Bayesian Block (BB) algorithm is a method specifically developed to bin gammaray light curves in blocks of time during which the rate varies within its Poisson uncertainty. An example of a BB light curve that uses the combined photon counts of the three selected NaI subdetectors is shown in Fig. 3. Long term fluctuations in the background rate imply that the transition to a new BB bin does not necessarily imply the appearance of a signal component. For each bin, the photon count is therefore compared to the expectation from the background characterisation. If a significant excess is observed, the BB light curves of the individual NaI detectors are examined to verify that the excess appears coincidently in at least two of the subdetectors before tagging it as a physical signal.

For bursts which were preceded by a precursor, the light curve will contain several periods of excess emission, separated by time intervals in which the rate returns to the background level ${ }^{2}$. This is illustrated in Fig. 3, where a precursor is observed $18 \mathrm{~s}$ before the start of the prompt phase. If a light curve contains multiple emission episodes, we tag the dominant excess, defined as the emission episode with the largest background subtracted photon count, as the prompt phase. All emission episodes that precede the prompt phase are accepted as precursors. In our analysis, a single GRB can thus have multiple precursors.

Before proceeding to the results, we first examine the probability that a random fluctuation would lead to a false precursor signal. A similar study [10], examining the data of 956 Fermi-GBM bursts, found only a single instance in which a burst had a precursor more than $500 \mathrm{~s}$ before the GBM trigger time. We therefore used the data from $1000 \mathrm{~s}$ to $500 \mathrm{~s}$ before the start of the Fermi-GBM trigger as a background control region. Based on the occurrence of emission episodes in this time window, we obtain a conservative estimate for the number of falsely identified precursor in our analysis of $36.1 \pm 8.8$.

\footnotetext{
${ }^{2}$ To avoid arbitrarily splitting a continuous emission episode due to a narrow low statistics bin in which a downward fluctuation causes the rate to drop down to or below the background expectation, an effective lower limit of $\sim 0.2 \mathrm{~s}$ is imposed on the duration of the required quiescent time.
} 




Figure 4: (top) Number of long GRBs that have a peak rate in excess of the value displayed on the $x$-axis. (bottom) Fraction of those GRBs that were preceded by at least one precursor. The grey band shows the $1 \sigma$ statistical uncertainty on this ratio.

\section{Results}

Following our selection criteria, which use a different method than the Fermi-GBM online trigger, 320 out of the 2684 GRBs did not show an emission excess. Restricting the analysis to the remaining 2364 bursts, a total of 244 precursor emission episodes were observed. The number of GRBs with a single, two and three precursors corresponds to 192, 23, and 2, respectively. No more than three precursors were observed for any single burst.

A major difference is observed between long and short GRBs. Only 1\% (4 out of 333) of short bursts have a precursor, versus $\sim 10 \%$ (213 out of 2031) of long bursts. In the case of long GRBs, the time separating the precursor from the prompt emission typically corresponds to a few tens of seconds. In contrast, all precursors to short GRBs occur within less than $2 \mathrm{~s}$ from the prompt emission. This latter observation is consistent with previous results $[3,20]$ and with model predictions for precursor signals from binary neutron star mergers [20-22].

Given that precursors are defined to be dimmer than the prompt emission, it is possible that, if a burst is located too far away, the precursor will be indistinguishable from the detector background. To mitigate this effect, previous precursor analyses [5-8] have sometimes restricted their GRB sample to 'sufficiently bright' long bursts. These studies did indeed also find the highest fraction of GRBs with precursors, up to $20 \%$ [5]. To examine the effect of such selection criteria on our analysis, we calculated the fraction of GRBs that have a precursor if only long GRBs with a peak flux above a certain threshold rate are considered. This relation is shown in Fig. 4. A clear increase is observed as a function of peak brightness, which reaches a plateau at $\sim 16 \%$. While a second even higher bump appears for the brightest bursts, this effect is statistically non-significant considering the number of bursts drastically decreases the higher the threshold rate, as evident from the top panel of Fig. 4. 


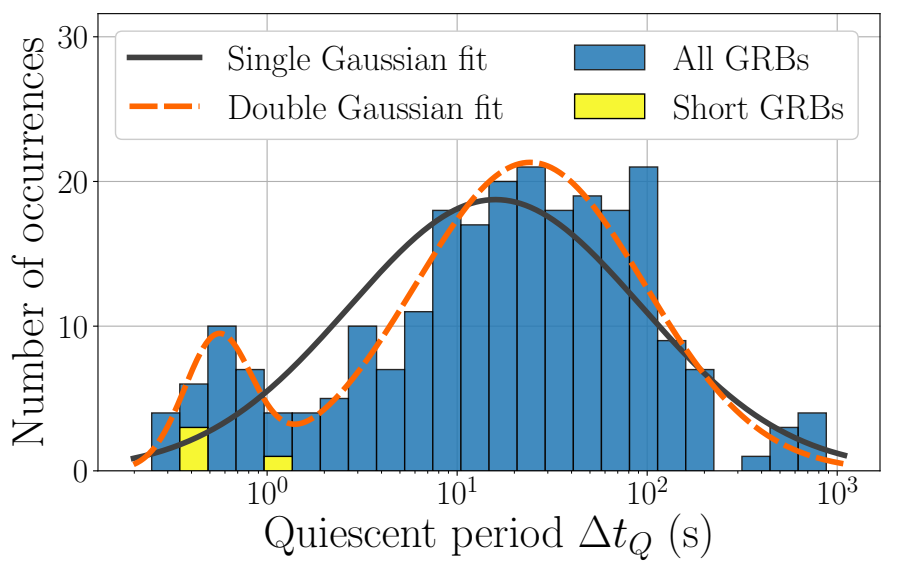

Figure 5: Quiescent times ${ }^{3}$ of the 244 identified precursors. A bimodal distribution is observed, indicating that the precursors of long GRBs could be caused by two different physical mechanisms.

As noted before, the precursors to short GRBs tend to occur much closer to the prompt phase than those of long GRBs. Examining the full distribution of the quiescent time ${ }^{3}$, shown in Fig. 5, illustrates this effect. A clear bimodality is moreover observed in the data. To quantify this claim, a single and double Gaussian fit are performed. Based on the best-fit parameters, the probability of obtaining a sample with a worse goodness-of-fit likelihood than the observed distribution was found to be $8 \cdot 10^{-5}$ and 0.36 for the single and double Gaussian fit, respectively. As such, the hypothesis that the data is consistent with a single Gaussian component can be rejected at $3.9 \sigma$. Focusing on the two component fit, the two peaks of the distribution are found to correspond to $(0.552 \pm 0.059) \mathrm{s}$ and $(24.2 \pm 1.2) \mathrm{s}$, with the first bump having a relative weight of $(11.1 \pm 2.6) \%$. These two bumps seems to indicate that the precursors of long GRBs can have different physical origins. Short time delays on the order of $\sim 1 \mathrm{~s}$ or less are expected from photospheric emission models [23], whereas longer delay precursors could be related to e.g. a reactivation of the central engine [11] or models that predict a jet-cocoon emission [24].

One burst of particular interest to the GRB community that showed two precursor signals in our analysis is GRB 190114C. This burst marked the first occasion that a GRB observation was reported with an imaging air Cherenkov telescope (IACT) and the first observation of $\mathrm{TeV}$-scale gamma rays from a GRB [25, 26]. Figure 6 displays the light curve of GRB $190114 \mathrm{C}$ as observed by the Fermi-GBM detector. Our analysis identified two precursors, indicated by the yellow bins, that occurred $5.57 \mathrm{~s}$ and $2.85 \mathrm{~s}$ prior to the start of the prompt emission and lasting for $1.94 \mathrm{~s}$ and $1.54 \mathrm{~s}$, respectively. While the excess can appear dim on Fig. 6 due to the range of the logarithmic $y$-axis, we note that the observed photon counts exceed the background expectation by a statistical significance of $6 \sigma$ in both cases. It thus appears that the production of gamma rays was already ongoing several seconds before the prompt emission was observed.

\footnotetext{
${ }^{3}$ The quiescent time is defined as the time duration between two emission episodes during which the rate returns to the background level.
} 


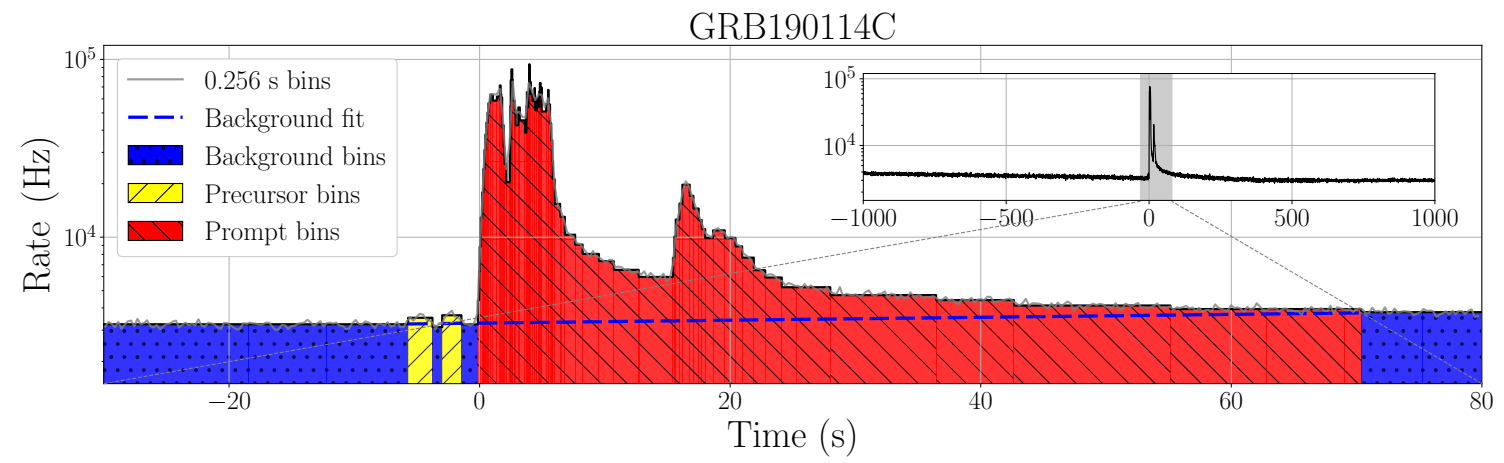

Figure 6: Light curve for GRB 190114C. Two precursors (yellow) are observed, starting $5.57 \mathrm{~s}$ and $2.85 \mathrm{~s}$ before the onset of the prompt (red) emission. The time range displayed in this figure corresponds to the grey shaded area in the inset image, which displays the light curve over a 2000 s period centred on the GBM trigger time.

\section{Conclusion}

Electromagnetic signals from gamma-ray bursts (GRBs) can be categorised into three observational stages: the precursor, prompt, and afterglow. Out of these three, the precursor phase is the least understood. We therefore performed an analysis on all GRBs detected by Fermi-GBM up to 2020 to identify and characterize precursor events. Out of a sample of 2364 bursts, 217 were observed to be preceded by one or multiple precursors. In terms of relative fractions, this corresponds to $\sim 1 \%$ of short GRBs and $\sim 10 \%$ of long GRBs having a precursor. A table containing all precursor emission times has been published in [16] and is, along with the Bayesian block light curves, available online [27]. One burst of particular interest which exhibited precursor emission is the ultra-bright and high-energy GRB 190114C.

Given that by definition, any precursor is required to be less bright than the prompt phase, precursors can be too weak to lead to a detectable signal, causing the true fraction of GRBs with a precursor to be underestimated. By restricting our analysis to long and bright bursts, we showed that this indeed is the case, suggesting that at least $\sim 16 \%$ of long GRBs have a precursor.

Looking at the time delay between different emission episodes, all precursors to short GRBs were found to occur within $2 \mathrm{~s}$ of the prompt emission. Precursors to long bursts, in contrast, were found to have a quiescent time ranging from less than a second up to several hundreds of seconds. The full distribution of the quiescent time was shown to be well described by a double Gaussian function, indicating that the precursors of long GRBs could be caused by two different physical mechanisms. For further details on the significance of this bimodal feature, along with the other results from the analysis, we refer the reader to [16].

\section{References}

[1] B. P. Abbott et al., ApJL 848 (Oct., 2017) L13.

[2] T. Murakami et al., Nature 350 (Apr., 1991) 592-594.

[3] E. Troja et al., ApJ 723 (oct, 2010) 1711-1717. 
[4] T. M. Koshut et al., ApJ 452 (Oct, 1995) 145.

[5] D. Lazzati, MNRAS 357 (Feb, 2005) 722-731.

[6] D. Burlon et al., A\&A 505 (Oct, 2009) 569-575.

[7] E. Ramirez-Ruiz et al., MNRAS 320 (Jan, 2001) L25-L29.

[8] L. Lan et al., ApJ 862 (Aug., 2018) 155.

[9] D. Burlon et al., ApJ 685 (Sep, 2008) L19.

[10] M. Charisi et al., MNRAS 448 (Apr, 2015) 2624-2633.

[11] Y.-D. Hu et al., ApJ 789 (Jul, 2014) 145.

[12] F. Nappo et al., MNRAS 445 (Dec, 2014) 1625-1635.

[13] P. Coppin et al. in ICRC2019, vol. 36, p. 859, July, 2019.

[14] G. Calderone et al. in The 7th INTEGRAL Workshop, p. 18, Jan, 2008.

[15] S.-Q. Zhong et al., ApJ 884 (Oct., 2019) 25.

[16] P. Coppin et al., PRD 102 (Nov., 2020) 103014.

[17] K. Deoskar et al., PoS(ICRC2021)469 (these proceedings).

[18] A. von Kienlin et al., ApJ 893 (Apr., 2020) 46.

[19] C. Meegan et al., ApJ 702 (Sep, 2009) 791-804.

[20] A. G. Suvorov et al., Phys. Rev. D 101 (Apr, 2020) 083002.

[21] B. D. Metzger et al., MNRAS 461 (Oct, 2016) 4435-4440.

[22] A. J. Penner et al., ApJ 749 (apr, 2012) L36.

[23] M. Lyutikov and V. V. Usov, ApJ 543 (Nov, 2000) L129-L132.

[24] D. Lazzati et al. in 22nd Texas Symposium on Relativistic Astrophysics, pp. 48-56, Jan., 2005.

[25] V. A. Acciari et al., Nature 575 (2019) 455-458.

[26] M. Ajello et al., ApJ 890 (Feb., 2020) 9.

[27] https://icecube.wisc.edu/ grbweb_public/Precursors.html. 